CRYSTALLOGRAPHIC COMMUNICATIONS

ISSN 2056-9890

Received 20 September 2021

Accepted 3 October 2021

Edited by A. V. Yatsenko, Moscow State University, Russia

Keywords: crystal structure; Schiff base; enolimine tautomer; $\mathrm{C}-\mathrm{H} \cdots \pi$ interactions; Hirshfeld surface analysis.

CCDC reference: 2113562

Supporting information: this article has supporting information at journals.iucr.org/e

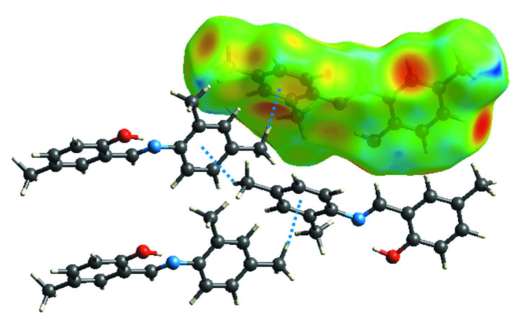

OPEN $\odot$ ACCESS

\section{Crystal structure and Hirshfeld surface analysis of (Z)-2-\{[(2,4-dimethylphenyl)imino]methyl\}-4- methylphenol}

\author{
Sevgi Kansiz, ${ }^{\mathrm{a} *}$ Adem Gul, $^{\mathrm{b}}$ Necmi Dege, ${ }^{\mathrm{c}}{\text { Erbil } \text { Agar }^{\mathrm{d}} \text { and Eiad Saif }}^{\mathrm{e}, \mathrm{f}_{*}}$
}

${ }^{\mathbf{a}}$ Samsun University, Faculty of Engineering, Department of Fundamental Sciences, 55420, Samsun, Turkey, bakay Pharma Medicine and Health Products Industries \& Trade, 34000, Istanbul, Turkey, ${ }^{\mathbf{C}}$ Ondokuz Mayıs University, Faculty of Arts and Sciences, Department of Physics, 55139, Samsun, Turkey, 'ondokuz Mayıs University, Faculty of Arts and Sciences, Department of Chemistry, 55139, Samsun, Turkey, ${ }^{\mathbf{e}}$ Department of Computer and Electronic Engineering Technology, Sanaa Community College, Sanaa, Yemen, and ${ }^{\mathbf{f}}$ Department of Electrical and Electronic Engineering, Faculty of Engineering, Ondokuz Mayıs University, 55139, Samsun, Turkey. *Correspondence e-mail:

sevgi.kansiz@samsun.edu.tr, eiad.saif@scc.edu.ye

The title compound, $\mathrm{C}_{16} \mathrm{H}_{17} \mathrm{NO}$, is a Schiff base that exists in the enol-imine tautomeric form and adopts a $Z$ configuration. The molecule is non-planar, with the twisted rings making a dihedral angle of $39.92(4)^{\circ}$. The intramolecular $\mathrm{O}-$ $\mathrm{H} \cdots \mathrm{N}$ hydrogen bond forms an $S(6)$ ring motif. In the crystal, molecules are linked by $\mathrm{C}-\mathrm{H} \cdots \pi$ interactions and very weak $\pi-\pi$ stacking interactions also help to consolidate the crystal packing. A Hirshfeld surface analysis was performed to investigate the contributions of different intermolecular contacts within the supramolecular structure. The major contributions are from $\mathrm{H} \cdots \mathrm{H}$ $(65 \%), \mathrm{C} \cdot \mathrm{H}(19.2 \%)$ and $\mathrm{O} \cdots \mathrm{H}(6.6 \%)$ interactions.

\section{Chemical context}

Schiff bases are well-known organic compounds widely used in many areas. These compounds can be easily synthesized by condensation of a primary aliphatic or aromatic amine with an aldehyde or ketone in different solvent media and they can easily be purified, since the amount of by-products is negligible (Tanak et al., 2020). Schiff bases are in general more stable than the compounds from which they are synthesized (Wadher et al., 2009). Nowadays, the possibility of molecular design is an important key for many research areas such as medicine or agriculture. In this respect, Schiff base formation provides an easy way to design new compounds, and biologically or chemically active compounds can be obtained using this method. As the structures of Schiff bases are generally similar to those of biological molecules, Schiff bases are valuable for understanding biological phenomena. As a result, Schiff bases are used in many studies. Various types of aldehydes or ketones have been used for their synthesis, but 2-hydroxybenzaldehyde and its derivatives are used especially often (Jeewoth et al., 2000; Mazhar et al., 2020). The basis of such preference is the tautomerism and stability provided by the hydroxyl group in conjunction with the imine group. Schiff bases with intramolecular hydrogen bonds can exhibit photochromic and thermochromic properties (Elerman et al., 2002). Schiff bases obtained from 2-hydroxybenzaldehyde and its derivatives can also form complexes with various metal ions. The title compound is a Schiff base prepared from 2-hydroxy-5-methylbenzaldehyde. 


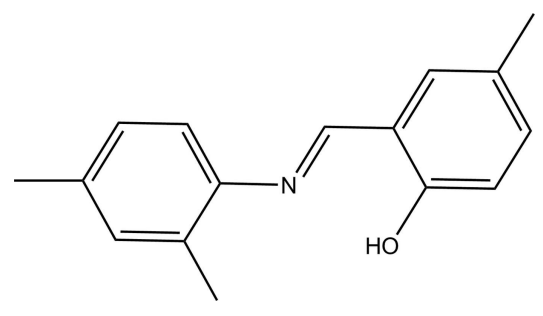

\section{Structural commentary}

The title compound crystallizes in the phenol-imine tautomeric form with an $Z$ configuration with respect to the imine bond. The asymmetric unit contains one molecule (Fig. 1), which is non-planar, two aromatic rings being twisted with respect to each other, subtending a dihedral angle of $39.92(4)^{\circ}$. The hydroxy and imine groups are involved in a strong intramolecular $\mathrm{O} 1-\mathrm{H} 1 \cdots \mathrm{N} 1$ hydrogen bond forming an $S(6)$ ring motif. The $\mathrm{C} 1-\mathrm{O} 1[1.353(2) \AA]$ and $\mathrm{C} 7-\mathrm{N} 1$ [1.282 (2) $\AA]$ bond distances indicate their single- and doublebond characters, respectively, being consistent with the phenol-imine tautomeric form.

\section{Supramolecular features}

In the crystal, molecules are linked by $\mathrm{C} 16-\mathrm{H} 16 A \cdots \pi$ (C9C14) interactions (Table 1, Fig. 2), and very weak $\pi-\pi$ stacking interactions between the $\mathrm{OH}$-substituted rings (C1-C6) related by the $a$ glide plane $\left[C g \cdots C g\left(-\frac{1}{2}+x, y, \frac{1}{2}-z\right)=\right.$ 4.0220 (9) $\AA$ ] lead to additional stabilization of the crystal packing. A view of the crystal packing parallel to the $b c$ plane is shown in Fig. 2.

\section{Database survey}

A search of the Cambridge Structural Database (CSD, version 5.42, update of May 2021; Groom et al., 2016) for the (Z)-2$\{[(2,4-$ dimethylphenyl)imino $]$ methyl $\}-4-m e t h y l p h e n o l ~ u n i t$, revealed ten hits where this fragment adopts the enol-imine tautomeric form. The imine bond length $(\mathrm{N} 1-\mathrm{C} 7)$ in the title compound is the same within standard uncertainties

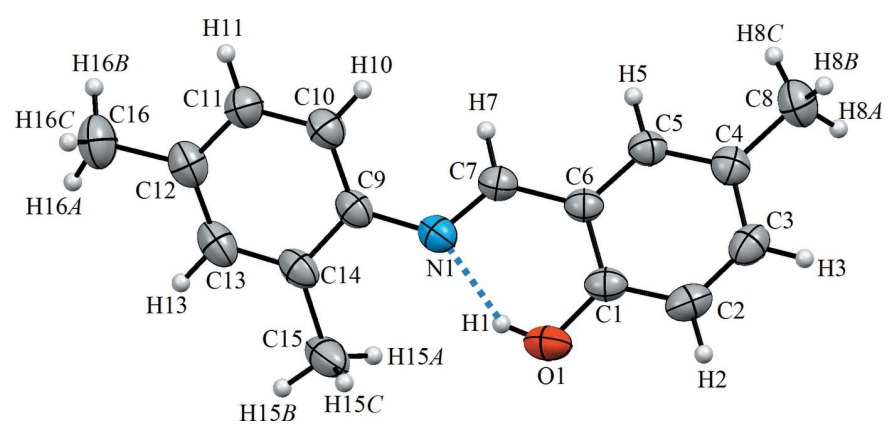

Figure 1

The molecular structure of the title compound, with atom labelling. Displacement ellipsoids are drawn at the $40 \%$ probability level. Dashed lines denote the intramolecular $\mathrm{O}-\mathrm{H} \cdots \mathrm{N}$ hydrogen bond forming an $S(6)$ ring motif.
Table 1

Hydrogen-bond geometry $\left(\AA,^{\circ}\right)$.

$\mathrm{Cg} 2$ is the centroid of the $\mathrm{C} 9-\mathrm{C} 14$ ring.

\begin{tabular}{lllll}
\hline$D-\mathrm{H} \cdots A$ & $D-\mathrm{H}$ & $\mathrm{H} \cdots A$ & $D \cdots A$ & $D-\mathrm{H} \cdots A$ \\
\hline $\mathrm{O} 1-\mathrm{H} 1 \cdots \mathrm{N} 1$ & 0.82 & 1.89 & $2.618(2)$ & 147 \\
$\mathrm{C} 16-\mathrm{H} 16 A \cdots C g 2^{\mathrm{i}}$ & 0.96 & $2.93(3)$ & 3.73 & 143 \\
\hline
\end{tabular}

Symmetry code: (i) $x+\frac{1}{2},-y+\frac{1}{2},-z$.

as the corresponding bond lengths in the structures of 2-(diphenylmethyl)-6-[(mesitylimino)methyl]-4-methylphenol (DEHQIS; Zhou et al., 2012), (R)- $N, N^{\prime}$-bis(3,5-di-t-butylsalicylidene)-5, $5^{\prime}, 6,6^{\prime}, 7,7^{\prime}, 8,8^{\prime}$-octahydro-1,1'-binaphthyl-2,2'diamine (MIFXAA; Jia et al., 2002), acetonitrile-bis $\{2-$ (mesitylcarbonoimidoyl)-6-[(mesitylimino)methyl]-4-methylphenolato\}magnesium acetonitrile solvate (QUDZAS; Ghosh et al., 2015), bis\{2,4-di-t-butyl-6-[(mesitylimino)methyl]phenolato\}tetrahydrofuranmagnesium (QUDZIA; Ghosh et al., 2015) and 2,4-di-t-butyl-6-\{[(2,4,6-tri-t-butylphenyl)imino]methyl\}phenol (YADZOV; Ma et al., 2016). As for the C1O1 bond $[1.353(2) \AA]$, its length compares well with 1.352 (2) $\AA$ for YADZOV and 1.359 (5) $\AA$ for DEHQIS. All other bond dimensions in the title structure agree well with those in previous literature reports. In NUGWES, NUGWIW and NUGWOC (Xu et al., 2009) and in YADZOV (Ma et al., 2016), the lengths of intramolecular $\mathrm{O}-\mathrm{H} \cdots \mathrm{N}$ hydrogen bonds are especially short, being within the range $1.81-1.88 \AA$.

\section{Hirshfeld surface analysis}

We have performed a Hirshfeld surface analysis and generated the associated two-dimensional fingerprint plots (Spackman \& Jayatilaka, 2009) with CrystalExplorer17 (Turner et al., 2017). Hirshfeld surface analysis is an important way of determining the location of atoms with potential to form hydrogen bonds and other intermolecular contacts, and the quantitative ratio of these interactions (Demircioğlu et al., 2019). The Hirshfeld

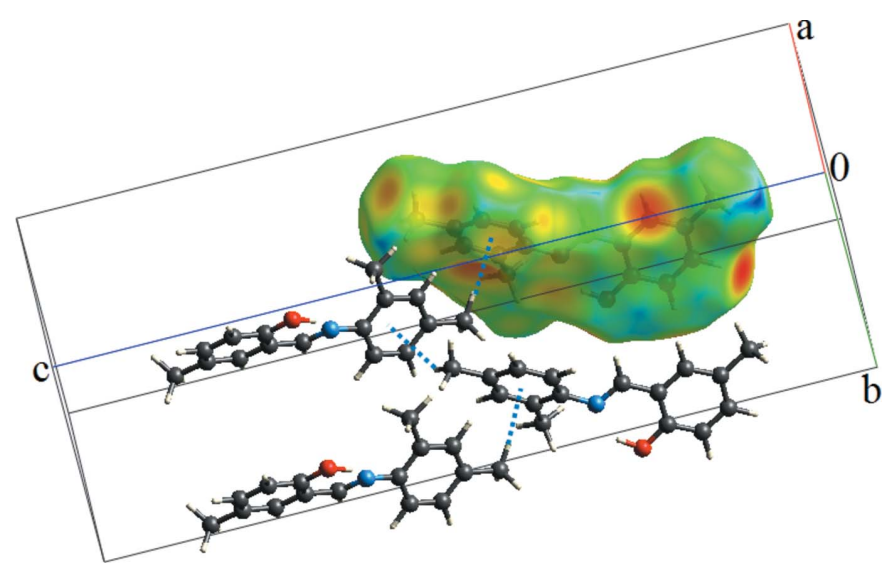

Figure 2

A view of the crystal packing of the title compound. The C16$\mathrm{H} 16 \mathrm{~A} \cdots \mathrm{Cg} 2$ interactions are denoted as dashed lines and as a red spot on the $d_{e}$ surface. 

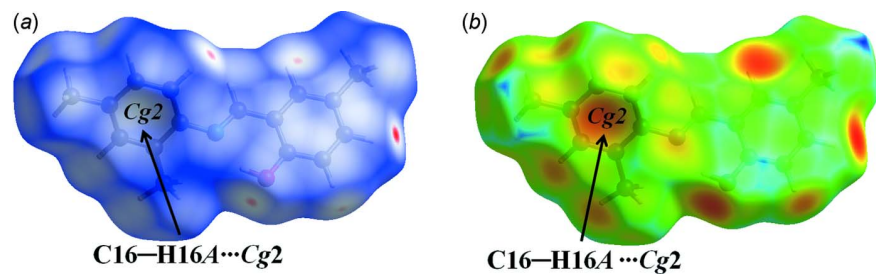

Figure 3

The red spots on the $d_{\text {norm }}$ and $d_{e}$ surfaces of the title molecule represent the $\mathrm{C}-\mathrm{H} \cdots \pi$ interactions.

surface was generated using a standard (high) surface resolution with the three-dimensional $d_{\text {norm }}$ surface mapped over a fixed colour scale of -0.1168 (red) to $1.1632 \AA$ (blue) (the fixed colour scale is 1.0201 to $2.4894 \AA$ for the $d_{e}$ surface). In Figs. 2 and 3 , the red spots on the $d_{\text {norm }}$ and $d_{e}$ surfaces represent the $\mathrm{C}-\mathrm{H} \cdots \mathrm{Cg}$ interactions. The most important interaction is $\mathrm{H} \cdots \mathrm{H}$, contributing $65 \%$ to the overall crystal packing, which is illustrated in the $2 \mathrm{D}$ fingerprint (Fig. 4). Two symmetrical wings on the left and right sides are seen in the fingerprint plot for $\mathrm{C} \cdots \mathrm{H} / \mathrm{H} \cdots \mathrm{C}$ interactions, the second most important contributor to the total Hirshfeld surface (19\%). The $\mathrm{O} \cdots \mathrm{H} / \mathrm{H} \cdots \mathrm{O}$ interactions provide a $6.6 \%$ contribution to the total Hirshfeld surface. Much weaker C...C $(5.3 \%)$, $\mathrm{N} \cdots \mathrm{H} / \mathrm{H} \cdots \mathrm{N}(2.3 \%)$ and $\mathrm{C} \cdots \mathrm{O} / \mathrm{O} \cdots \mathrm{C}(1.3 \%)$ contacts are also present.

\section{Synthesis and crystallization}

(Z)-2-\{[(2,4-dimethylphenyl)imino]methyl\}-4-methylphenol was synthesized by condensation of 2-hydroxy-5-methylbenzaldehyde and 2,4-dimethylaniline (Fig. 5). For this
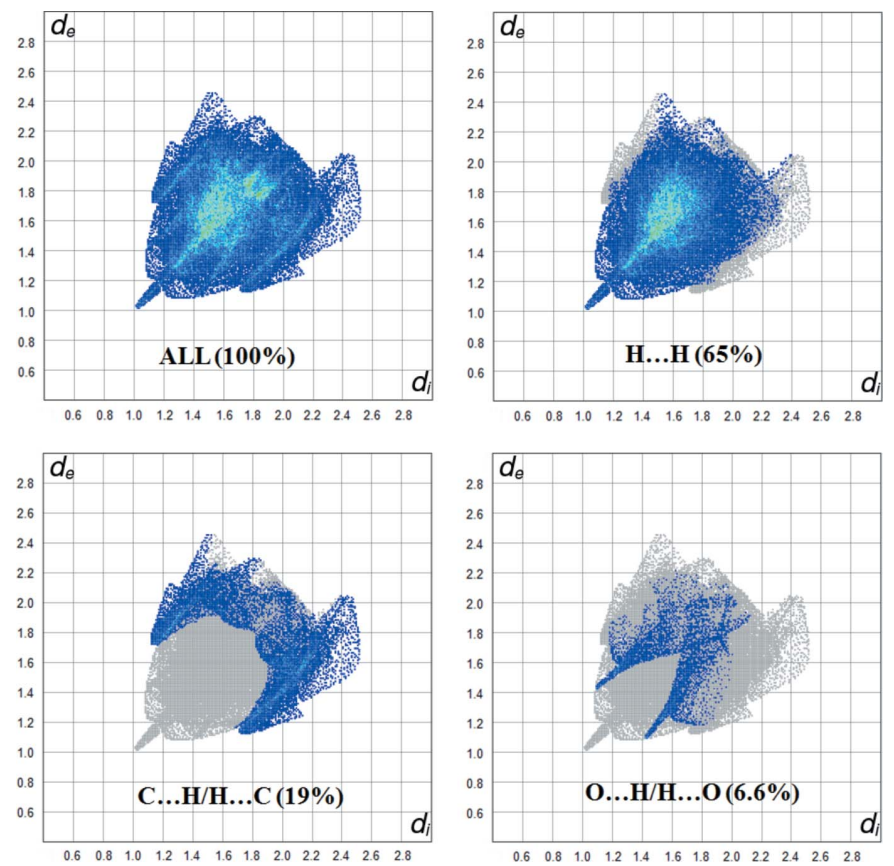

Figure 4

Fingerprint plots showing all intermolecular interactions and resolved into $\mathrm{H} \cdots \mathrm{H}, \mathrm{C} \cdots \mathrm{H} / \mathrm{H} \cdots \mathrm{C}$ and $\mathrm{O} \cdots \mathrm{H} / \mathrm{H} \cdots \mathrm{O}$ contacts.

Table 2

Experimental details.

Crystal data

Chemical formula

$M_{\text {r }}$

Crystal system, space group

Temperature $(\mathrm{K})$

$a, b, c(\AA)$

$V\left(\AA^{3}\right)$

$Z$

Radiation type

$\mu\left(\mathrm{mm}^{-1}\right)$

Crystal size (mm)

Data collection

Diffractometer

Absorption correction

$T_{\min }, T_{\max }$

No. of measured, independent and observed $[I>2 \sigma(I)]$ reflections

$R_{\text {int }}$

$(\sin \theta / \lambda)_{\max }\left(\AA^{-1}\right)$

Refinement

$R\left[F^{2}>2 \sigma\left(F^{2}\right)\right], w R\left(F^{2}\right), S$

No. of reflections

No. of parameters

H-atom treatment

$\Delta \rho_{\max }, \Delta \rho_{\min }\left(\mathrm{e} \AA^{-3}\right)$

Computer programs: $X$-AREA (Stoe \& Cie, 2002), $X$-RED (Stoe \& Cie, 2002), SHELXT2017/1 (Sheldrick, 2015a), SHELXL2017/1 (Sheldrick, 2015b), PLATON (Spek, 2020), WinGX (Farrugia, 2012).

purpose, a mixture of a solution containing 2-hydroxy-5methylbenzaldehyde $(0.04 \mathrm{mmol})$ in ethanol $(20 \mathrm{~mL})$ and a solution containing 2,4-dimethylaniline $(0.04 \mathrm{mmol})$ in ethanol $(20 \mathrm{~mL})$ was refluxed for $6 \mathrm{~h}$ under stirring. The obtained crystalline product was washed with ethanol and dried at room temperature. Single crystals were obtained by slow evaporation of ethanol solution at room temperature.

\section{Refinement}

Crystal data, data collection and structure refinement details are summarized in Table 2. The $\mathrm{O}$-bound $\mathrm{H}$ atom was located in a difference-Fourier map and refined with $\mathrm{O}-\mathrm{H}=0.82 \AA$, and with $U_{\text {iso }}(\mathrm{H})=1.5 U_{\text {eq }}(\mathrm{O})$. The C-bound $\mathrm{H}$ atoms were positioned geometrically and refined using a riding model with $\mathrm{C}-\mathrm{H}=0.93$ and $U_{\text {iso }}(\mathrm{H})=1.2 U_{\text {eq }}(\mathrm{C})$ for $s p^{2}$-hybridized $\mathrm{C}$ atoms and with $\mathrm{C}-\mathrm{H}=0.96 \AA$ and $U_{\text {iso }}(\mathrm{H})=1.5 U_{\text {eq }}(\mathrm{C})$ for methyl groups.

\section{Acknowledgements}

Author contributions are as follows. Conceptualization, SK, ND and ES; synthesis, AG and EA; writing (review and

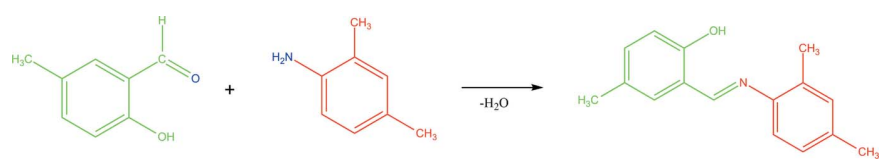

Figure 5

The scheme of synthesis of the title compound. 
editing of the manuscript) SK, EA and AG; formal analysis, SK and ND; crystal-structure determination, ND; validation, SK, ND and EA; project administration, SK, ND and ES.

\section{Funding information}

This study was supported by Ondokuz Mayıs University under Project No. PYO·FEN.1906.19.001.

\section{References}

Demircioğlu, Z., Kaştaş, G., Kaştaş, Ç. A. \& Frank, R. (2019). J. Mol. Struct. 1191, 129-137.

Elerman, Y., Kabak, M., Elmali, A. \& Naturforsch, Z. B. (2002). Chem. Sci. 57, 651-656.

Farrugia, L. J. (2012). J. Appl. Cryst. 45, 849-854.

Ghosh, S., Chakraborty, D. \& Ramkumar, V. (2015). J. Polym. Sci. Part A Polym. Chem. 53, 1474-1491.

Groom, C. R., Bruno, I. J., Lightfoot, M. P. \& Ward, S. C. (2016). Acta Cryst. B72, 171-179.

Jeewoth, T., Li Kam Wah, H., Bhowon, M. G., Ghoorohoo, D. \& Babooram, K. (2000). Synth. React. Inorg. Met.-Org. Chem. 30, 1023-1038.
Jia, X., Li, X. \& Zhou, Z. (2002). Acta Cryst. E58, o183-o184.

Ma, M., Shen, X., Wang, W., Li, J., Yao, W. \& Zhu, L. (2016). Eur. J. Inorg. Chem. 2016, 5057-5062.

Mazhar, N., Aftab, M., Mahmud, T., Basra, M. R., Akhtar, M. \& Mitu, L. (2020). Rev. Chim. 71, 47-58.

Sheldrick, G. M. (2015a). Acta Cryst. A71, 3-8.

Sheldrick, G. M. (2015b). Acta Cryst. C71, 3-8.

Spackman, M. A. \& Jayatilaka, D. (2009). CrystEngComm, 11, 19-32. Spek, A. L. (2020). Acta Cryst. E76, 1-11.

Stoe \& Cie (2002). $X$-AREA and $X$-RED32. Stoe \& Cie GmbH, Darmstadt, Germany.

Tanak, H., Karataş, Ş., Meral, S. \& Ağar, E. (2020). Crystallogr. Rep. $\mathbf{6 5}, 1221-1225$.

Turner, M. J., MacKinnon, J. J., Wolff, S. K., Grimwood, D. J., Spackman, P. R., Jayatilaka, D. \& Spackman, M. A. (2017). Crystal Explorer 17.5. University of Western Australia. http://hirshfeldsurface.net.

Wadher, S. J., Puranik, M. P., Karande, N. A. \& Yeole, P. G. (2009). Int. J. Pharmtech Res. 1, 22-33.

Xu, Z.-X., Huang, Z.-T. \& Chen, C.-F. (2009). Tetrahedron Lett. 50, 5430-5433.

Zhou, Z., Li, W., Hou, X., Chen, L., Hao, X., Redshaw, C. \& Sun, W.-H. (2012). Inorg. Chim. Acta, 392, 292-299. 


\section{supporting information}

Acta Cryst. (2021). E77, 1087-1090 [https://doi.org/10.1107/S2056989021010215]

Crystal structure and Hirshfeld surface analysis of (Z)-2-\{[(2,4-dimethylphenyl)imino]methyl\}-4-methylphenol

Sevgi Kansiz, Adem Gul, Necmi Dege, Erbil Agar and Eiad Saif

Computing details

Data collection: $X$-AREA (Stoe \& Cie, 2002); cell refinement: $X$-AREA (Stoe \& Cie, 2002); data reduction: $X$-RED (Stoe \& Cie, 2002); program(s) used to solve structure: SHELXT2017/1 (Sheldrick, 2015a); program(s) used to refine structure: SHELXL2017/1 (Sheldrick, 2015b); molecular graphics: PLATON (Spek, 2020); software used to prepare material for publication: $\operatorname{Win} G X$ (Farrugia, 2012).

(Z)-2-\{[(2,4-Dimethylphenyl)imino]methyl\}-4-methylphenol

Crystal data

\section{$\mathrm{C}_{16} \mathrm{H}_{17} \mathrm{NO}$}

$M_{r}=239.30$

Orthorhombic, $\mathrm{Pbca}$

$a=7.6699$ (4) $\AA$

$b=11.6080(6) \AA$

$c=30.1431(17) \AA$

$V=2683.7(2) \AA^{3}$

$Z=8$

$F(000)=1024$

Data collection

Stoe IPDS 2 diffractometer

Radiation source: sealed X-ray tube, 12 x 0.4 $\mathrm{mm}$ long-fine focus

Detector resolution: 6.67 pixels $\mathrm{mm}^{-1}$ rotation method scans

Absorption correction: integration (X-RED32; Stoe \& Cie, 2002)

$T_{\min }=0.953, T_{\max }=0.990$

\section{Refinement}

Refinement on $F^{2}$

Least-squares matrix: full

$R\left[F^{2}>2 \sigma\left(F^{2}\right)\right]=0.042$

$w R\left(F^{2}\right)=0.125$

$S=1.00$

2213 reflections

168 parameters

0 restraints
$D_{\mathrm{x}}=1.185 \mathrm{Mg} \mathrm{m}^{-3}$

Mo $K \alpha$ radiation, $\lambda=0.71073 \AA$

Cell parameters from 13620 reflections

$\theta=1.4-24.9^{\circ}$

$\mu=0.07 \mathrm{~mm}^{-1}$

$T=296 \mathrm{~K}$

Plate, orange

$0.68 \times 0.48 \times 0.18 \mathrm{~mm}$

14869 measured reflections

2213 independent reflections

1451 reflections with $I>2 \sigma(I)$

$R_{\text {int }}=0.081$

$\theta_{\max }=24.5^{\circ}, \theta_{\min }=1.4^{\circ}$

$h=-8 \rightarrow 8$

$k=-13 \rightarrow 13$

$l=-34 \rightarrow 34$

Primary atom site location: structure-invariant direct methods

Hydrogen site location: inferred from neighbouring sites

$\mathrm{H}$-atom parameters constrained

$w=1 /\left[\sigma^{2}\left(F_{\mathrm{o}}{ }^{2}\right)+(0.0725 P)^{2}\right]$

where $P=\left(F_{\mathrm{o}}^{2}+2 F_{\mathrm{c}}^{2}\right) / 3$

$(\Delta / \sigma)_{\max }<0.001$

$\Delta \rho_{\max }=0.11$ e $\AA^{-3}$ 
$\Delta \rho_{\min }=-0.11$ e $\AA^{-3}$
Extinction correction:

file://iucrfs/e/yk2157/yk2157.cif, $\mathrm{Fc}^{*}=\mathrm{kFc}\left[1+0.001 \mathrm{xFc}^{2} \lambda^{3} / \sin (2 \theta)\right]^{-1 / 4}$

Extinction coefficient: 0.0094 (14)

Special details

Geometry. All esds (except the esd in the dihedral angle between two 1.s. planes) are estimated using the full covariance matrix. The cell esds are taken into account individually in the estimation of esds in distances, angles and torsion angles; correlations between esds in cell parameters are only used when they are defined by crystal symmetry. An approximate (isotropic) treatment of cell esds is used for estimating esds involving l.s. planes.

Fractional atomic coordinates and isotropic or equivalent isotropic displacement parameters $\left(\AA^{2}\right)$

\begin{tabular}{|c|c|c|c|c|}
\hline & $x$ & $y$ & $z$ & $U_{\text {iso }} * / U_{\text {eq }}$ \\
\hline $\mathrm{O} 1$ & $0.3099(2)$ & $0.58146(10)$ & $0.30727(4)$ & $0.0844(4)$ \\
\hline H1 & 0.330751 & 0.540427 & 0.328769 & $0.127 *$ \\
\hline N1 & $0.42492(18)$ & $0.40012(12)$ & $0.34984(5)$ & 0.0663 (4) \\
\hline C6 & $0.4130(2)$ & 0.40987 (13) & $0.27056(6)$ & 0.0569 (4) \\
\hline $\mathrm{C} 5$ & 0.4477 (2) & $0.35487(14)$ & $0.23014(5)$ & $0.0595(4)$ \\
\hline H5 & 0.495649 & 0.281288 & 0.230684 & $0.071 *$ \\
\hline C7 & $0.4516(2)$ & $0.35261(15)$ & $0.31204(6)$ & $0.0611(5)$ \\
\hline $\mathrm{H} 7$ & 0.497415 & 0.278473 & 0.311267 & $0.073 *$ \\
\hline $\mathrm{C} 1$ & $0.3426(2)$ & $0.52223(13)$ & $0.26953(6)$ & $0.0630(5)$ \\
\hline C9 & $0.4629(2)$ & $0.33967(15)$ & $0.38970(6)$ & $0.0633(5)$ \\
\hline $\mathrm{C} 4$ & 0.4139 (2) & $0.40502(16)$ & $0.18961(6)$ & $0.0648(5)$ \\
\hline C14 & $0.5297(2)$ & $0.40228(16)$ & $0.42555(6)$ & $0.0692(5)$ \\
\hline $\mathrm{C} 10$ & $0.4302(2)$ & $0.22231(16)$ & $0.39438(6)$ & $0.0724(5)$ \\
\hline H10 & 0.383752 & 0.180914 & 0.370760 & $0.087 *$ \\
\hline $\mathrm{C} 2$ & 0.3077 (2) & $0.57301(15)$ & $0.22917(7)$ & 0.0739 (5) \\
\hline $\mathrm{H} 2$ & 0.259857 & 0.646596 & 0.228269 & $0.089 *$ \\
\hline $\mathrm{C} 3$ & $0.3428(2)$ & $0.51607(16)$ & $0.19032(7)$ & $0.0736(5)$ \\
\hline H3 & 0.318475 & 0.552460 & 0.163539 & $0.088^{*}$ \\
\hline $\mathrm{C} 12$ & $0.5363(2)$ & $0.22599(19)$ & $0.46974(6)$ & $0.0759(6)$ \\
\hline $\mathrm{C} 13$ & $0.5668(2)$ & $0.34303(19)$ & $0.46426(6)$ & $0.0771(6)$ \\
\hline H13 & 0.614731 & 0.383787 & 0.487863 & $0.093 *$ \\
\hline C11 & $0.4663(3)$ & $0.16695(17)$ & $0.43391(6)$ & $0.0774(5)$ \\
\hline H11 & 0.443232 & 0.088594 & 0.436544 & $0.093 *$ \\
\hline C8 & $0.4528(3)$ & $0.34410(19)$ & $0.14659(6)$ & $0.0893(6)$ \\
\hline H8A & 0.524423 & 0.392488 & 0.128285 & $0.134 *$ \\
\hline H8B & 0.345600 & 0.327600 & 0.131447 & $0.134^{*}$ \\
\hline $\mathrm{H} 8 \mathrm{C}$ & 0.513250 & 0.273397 & 0.152645 & $0.134^{*}$ \\
\hline C15 & $0.5618(3)$ & $0.53050(18)$ & $0.42172(7)$ & $0.0970(7)$ \\
\hline H15A & 0.638745 & 0.545186 & 0.397253 & $0.145^{*}$ \\
\hline H15B & 0.613945 & 0.558242 & 0.448631 & $0.145^{*}$ \\
\hline $\mathrm{H} 15 \mathrm{C}$ & 0.453020 & 0.569432 & 0.416857 & $0.145^{*}$ \\
\hline C16 & $0.5729(3)$ & 0.1666 (2) & $0.51352(7)$ & $0.1014(7)$ \\
\hline H16A & 0.650363 & 0.213308 & 0.530910 & $0.152 *$ \\
\hline H16B & 0.625932 & 0.093075 & 0.508055 & $0.152^{*}$ \\
\hline H16C & 0.465487 & 0.155857 & 0.529352 & $0.152^{*}$ \\
\hline
\end{tabular}


Atomic displacement parameters $\left(\AA^{2}\right)$

\begin{tabular}{lllllll}
\hline & $U^{11}$ & $U^{22}$ & $U^{33}$ & $U^{12}$ & $U^{13}$ & $U^{23}$ \\
\hline O1 & $0.0935(10)$ & $0.0608(7)$ & $0.0990(10)$ & $0.0078(7)$ & $0.0082(9)$ & $-0.0177(7)$ \\
N1 & $0.0658(9)$ & $0.0681(9)$ & $0.0649(10)$ & $-0.0063(7)$ & $0.0056(8)$ & $-0.0106(8)$ \\
C6 & $0.0514(9)$ & $0.0516(9)$ & $0.0676(10)$ & $-0.0051(7)$ & $-0.0008(8)$ & $-0.0042(8)$ \\
C5 & $0.0546(9)$ & $0.0567(9)$ & $0.0673(11)$ & $-0.0019(7)$ & $-0.0042(9)$ & $-0.0018(9)$ \\
C7 & $0.0588(10)$ & $0.0572(10)$ & $0.0672(11)$ & $-0.0014(8)$ & $0.0001(9)$ & $-0.0053(9)$ \\
C1 & $0.0544(9)$ & $0.0532(9)$ & $0.0813(12)$ & $-0.0049(7)$ & $0.0020(10)$ & $-0.0063(10)$ \\
C9 & $0.0585(10)$ & $0.0731(12)$ & $0.0582(10)$ & $-0.0035(8)$ & $0.0057(9)$ & $-0.0098(9)$ \\
C4 & $0.0552(10)$ & $0.0704(12)$ & $0.0686(12)$ & $-0.0072(8)$ & $-0.0058(9)$ & $0.0003(9)$ \\
C14 & $0.0639(11)$ & $0.0820(12)$ & $0.0618(11)$ & $-0.0096(9)$ & $0.0138(9)$ & $-0.0186(10)$ \\
C10 & $0.0748(12)$ & $0.0757(12)$ & $0.0667(12)$ & $-0.0056(9)$ & $-0.0078(10)$ & $-0.0093(9)$ \\
C2 & $0.0638(11)$ & $0.0548(10)$ & $0.1032(15)$ & $-0.0006(8)$ & $-0.0073(11)$ & $0.0066(11)$ \\
C3 & $0.0672(12)$ & $0.0713(12)$ & $0.0822(14)$ & $-0.0090(10)$ & $-0.0129(10)$ & $0.0163(10)$ \\
C12 & $0.0647(11)$ & $0.0989(15)$ & $0.0642(12)$ & $0.0014(10)$ & $-0.0002(9)$ & $-0.0072(10)$ \\
C13 & $0.0672(12)$ & $0.1049(16)$ & $0.0593(11)$ & $-0.0127(11)$ & $0.0051(9)$ & $-0.0186(10)$ \\
C11 & $0.0842(13)$ & $0.0762(12)$ & $0.0716(12)$ & $0.0001(10)$ & $-0.0075(11)$ & $-0.0006(10)$ \\
C8 & $0.0923(15)$ & $0.1077(16)$ & $0.0680(13)$ & $0.0019(12)$ & $-0.0081(11)$ & $-0.0041(11)$ \\
C15 & $0.1220(19)$ & $0.0904(15)$ & $0.0785(13)$ & $-0.0314(13)$ & $0.0196(13)$ & $-0.0273(11)$ \\
C16 & $0.1019(17)$ & $0.130(2)$ & $0.0727(14)$ & $-0.0012(14)$ & $-0.0154(12)$ & $0.0056(13)$ \\
& & & & & & \\
\hline
\end{tabular}

Geometric parameters $\left(\AA,{ }^{\circ}\right)$

\begin{tabular}{llll}
\hline $\mathrm{O} 1-\mathrm{C} 1$ & $1.353(2)$ & $\mathrm{C} 10-\mathrm{H} 10$ & 0.9300 \\
$\mathrm{O} 1-\mathrm{H} 1$ & 0.8200 & $\mathrm{C} 2-\mathrm{C} 3$ & $1.371(3)$ \\
$\mathrm{N} 1-\mathrm{C} 7$ & $1.2823(19)$ & $\mathrm{C} 2-\mathrm{H} 2$ & 0.9300 \\
$\mathrm{~N} 1-\mathrm{C} 9$ & $1.421(2)$ & $\mathrm{C} 3-\mathrm{H} 3$ & 0.9300 \\
$\mathrm{C} 6-\mathrm{C} 5$ & $1.401(2)$ & $\mathrm{C} 12-\mathrm{C} 11$ & $1.387(3)$ \\
$\mathrm{C} 6-\mathrm{C} 1$ & $1.412(2)$ & $\mathrm{C} 12-\mathrm{C} 13$ & $1.388(3)$ \\
$\mathrm{C} 6-\mathrm{C} 7$ & $1.447(2)$ & $\mathrm{C} 12-\mathrm{C} 16$ & $1.515(3)$ \\
$\mathrm{C} 5-\mathrm{C} 4$ & $1.378(2)$ & $\mathrm{C} 13-\mathrm{H} 13$ & 0.9300 \\
$\mathrm{C} 5-\mathrm{H} 5$ & 0.9300 & $\mathrm{C} 11-\mathrm{H} 11$ & 0.9300 \\
$\mathrm{C} 7-\mathrm{H} 7$ & 0.9300 & $\mathrm{C} 8-\mathrm{H} 8 \mathrm{~A}$ & 0.9600 \\
$\mathrm{C} 1-\mathrm{C} 2$ & $1.378(2)$ & $\mathrm{C} 8-\mathrm{H} 8 \mathrm{~B}$ & 0.9600 \\
$\mathrm{C} 9-\mathrm{C} 10$ & $1.392(2)$ & $\mathrm{C} 8-\mathrm{H} 8 \mathrm{C}$ & 0.9600 \\
$\mathrm{C} 9-\mathrm{C} 14$ & $1.400(2)$ & $\mathrm{C} 15-\mathrm{H} 15 \mathrm{~A}$ & 0.9600 \\
$\mathrm{C} 4-\mathrm{C} 3$ & $1.400(3)$ & $\mathrm{C} 15-\mathrm{H} 15 \mathrm{~B}$ & 0.9600 \\
$\mathrm{C} 4-\mathrm{C} 8$ & $1.507(3)$ & $\mathrm{C} 15-\mathrm{H} 15 \mathrm{C}$ & 0.9600 \\
$\mathrm{C} 14-\mathrm{C} 13$ & $1.384(3)$ & $\mathrm{C} 16-\mathrm{H} 16 \mathrm{~A}$ & 0.9600 \\
$\mathrm{C} 14-\mathrm{C} 15$ & $1.513(3)$ & $\mathrm{C} 16-\mathrm{H} 16 \mathrm{~B}$ & 0.9600 \\
$\mathrm{C} 10-\mathrm{C} 11$ & $1.382(2)$ & $\mathrm{C} 16-\mathrm{H} 16 \mathrm{C}$ & 0.9600 \\
& & & 118.9 \\
$\mathrm{C} 1-\mathrm{O} 1-\mathrm{H} 1$ & 109.5 & $\mathrm{C} 2-\mathrm{C} 3-\mathrm{H} 3$ & 118.9 \\
$\mathrm{C} 7-\mathrm{N} 1-\mathrm{C} 9$ & $120.40(15)$ & $\mathrm{C} 4-\mathrm{C} 3-\mathrm{H} 3$ & $117.14(18)$ \\
$\mathrm{C} 5-\mathrm{C} 6-\mathrm{C} 1$ & $118.32(16)$ & $\mathrm{C} 11-\mathrm{C} 12-\mathrm{C} 13$ & $121.7(2)$ \\
$\mathrm{C} 5-\mathrm{C} 6-\mathrm{C} 7$ & $120.23(15)$ & $\mathrm{C} 11-\mathrm{C} 12-\mathrm{C} 16$ & $121.16(18)$ \\
$\mathrm{C} 1-\mathrm{C} 6-\mathrm{C} 7$ & $121.44(16)$ & $\mathrm{C} 13-\mathrm{C} 12-\mathrm{C} 16$ & \\
& & &
\end{tabular}




\begin{tabular}{|c|c|}
\hline $\mathrm{C} 4-\mathrm{C} 5-\mathrm{C} 6$ & $122.88(16)$ \\
\hline $\mathrm{C} 4-\mathrm{C} 5-\mathrm{H} 5$ & 118.6 \\
\hline $\mathrm{C} 6-\mathrm{C} 5-\mathrm{H} 5$ & 118.6 \\
\hline $\mathrm{N} 1-\mathrm{C} 7-\mathrm{C} 6$ & $122.54(16)$ \\
\hline $\mathrm{N} 1-\mathrm{C} 7-\mathrm{H} 7$ & 118.7 \\
\hline $\mathrm{C} 6-\mathrm{C} 7-\mathrm{H} 7$ & 118.7 \\
\hline $\mathrm{O} 1-\mathrm{C} 1-\mathrm{C} 2$ & $119.28(16)$ \\
\hline $\mathrm{O} 1-\mathrm{C} 1-\mathrm{C} 6$ & $121.45(17)$ \\
\hline $\mathrm{C} 2-\mathrm{C} 1-\mathrm{C} 6$ & $119.26(17)$ \\
\hline $\mathrm{C} 10-\mathrm{C} 9-\mathrm{C} 14$ & $119.71(17)$ \\
\hline $\mathrm{C} 10-\mathrm{C} 9-\mathrm{N} 1$ & $122.14(15)$ \\
\hline $\mathrm{C} 14-\mathrm{C} 9-\mathrm{N} 1$ & $118.12(16)$ \\
\hline $\mathrm{C} 5-\mathrm{C} 4-\mathrm{C} 3$ & $116.66(17)$ \\
\hline $\mathrm{C} 5-\mathrm{C} 4-\mathrm{C} 8$ & $121.83(17)$ \\
\hline $\mathrm{C} 3-\mathrm{C} 4-\mathrm{C} 8$ & $121.50(17)$ \\
\hline $\mathrm{C} 13-\mathrm{C} 14-\mathrm{C} 9$ & $117.93(17)$ \\
\hline $\mathrm{C} 13-\mathrm{C} 14-\mathrm{C} 15$ & $121.31(17)$ \\
\hline $\mathrm{C} 9-\mathrm{C} 14-\mathrm{C} 15$ & $120.76(18)$ \\
\hline $\mathrm{C} 11-\mathrm{C} 10-\mathrm{C} 9$ & $120.43(17)$ \\
\hline $\mathrm{C} 11-\mathrm{C} 10-\mathrm{H} 10$ & 119.8 \\
\hline $\mathrm{C} 9-\mathrm{C} 10-\mathrm{H} 10$ & 119.8 \\
\hline $\mathrm{C} 3-\mathrm{C} 2-\mathrm{C} 1$ & $120.63(17)$ \\
\hline $\mathrm{C} 3-\mathrm{C} 2-\mathrm{H} 2$ & 119.7 \\
\hline $\mathrm{C} 1-\mathrm{C} 2-\mathrm{H} 2$ & 119.7 \\
\hline $\mathrm{C} 2-\mathrm{C} 3-\mathrm{C} 4$ & $122.24(17)$ \\
\hline $\mathrm{C} 1-\mathrm{C} 6-\mathrm{C} 5-\mathrm{C} 4$ & $-0.9(2)$ \\
\hline $\mathrm{C} 7-\mathrm{C} 6-\mathrm{C} 5-\mathrm{C} 4$ & $-179.97(15)$ \\
\hline $\mathrm{C} 9-\mathrm{N} 1-\mathrm{C} 7-\mathrm{C} 6$ & $179.20(14)$ \\
\hline $\mathrm{C} 5-\mathrm{C} 6-\mathrm{C} 7-\mathrm{N} 1$ & $178.78(14)$ \\
\hline $\mathrm{C} 1-\mathrm{C} 6-\mathrm{C} 7-\mathrm{N} 1$ & $-0.3(2)$ \\
\hline $\mathrm{C} 5-\mathrm{C} 6-\mathrm{C} 1-\mathrm{O} 1$ & $-178.36(15)$ \\
\hline $\mathrm{C} 7-\mathrm{C} 6-\mathrm{C} 1-\mathrm{O} 1$ & $0.7(2)$ \\
\hline $\mathrm{C} 5-\mathrm{C} 6-\mathrm{C} 1-\mathrm{C} 2$ & $1.1(2)$ \\
\hline $\mathrm{C} 7-\mathrm{C} 6-\mathrm{C} 1-\mathrm{C} 2$ & $-179.83(15)$ \\
\hline $\mathrm{C} 7-\mathrm{N} 1-\mathrm{C} 9-\mathrm{C} 10$ & $-38.6(2)$ \\
\hline $\mathrm{C} 7-\mathrm{N} 1-\mathrm{C} 9-\mathrm{C} 14$ & $143.67(16)$ \\
\hline $\mathrm{C} 6-\mathrm{C} 5-\mathrm{C} 4-\mathrm{C} 3$ & $0.4(2)$ \\
\hline $\mathrm{C} 6-\mathrm{C} 5-\mathrm{C} 4-\mathrm{C} 8$ & $179.87(15)$ \\
\hline $\mathrm{C} 10-\mathrm{C} 9-\mathrm{C} 14-\mathrm{C} 13$ & $2.3(3)$ \\
\hline $\mathrm{N} 1-\mathrm{C} 9-\mathrm{C} 14-\mathrm{C} 13$ & $-179.87(15)$ \\
\hline $\mathrm{C} 10-\mathrm{C} 9-\mathrm{C} 14-\mathrm{C} 15$ & $-178.33(17)$ \\
\hline
\end{tabular}

$\mathrm{C} 14-\mathrm{C} 13-\mathrm{C} 12$
$\mathrm{C} 14-\mathrm{C} 13-\mathrm{H} 13$
$\mathrm{C} 12-\mathrm{C} 13-\mathrm{H} 13$
$\mathrm{C} 10-\mathrm{C} 11-\mathrm{C} 12$

$\mathrm{C} 10-\mathrm{C} 11-\mathrm{H} 11$

C12-C11-H11

$\mathrm{C} 4-\mathrm{C} 8-\mathrm{H} 8 \mathrm{~A}$

$\mathrm{C} 4-\mathrm{C} 8-\mathrm{H} 8 \mathrm{~B}$

$\mathrm{H} 8 \mathrm{~A}-\mathrm{C} 8-\mathrm{H} 8 \mathrm{~B}$

$\mathrm{C} 4-\mathrm{C} 8-\mathrm{H} 8 \mathrm{C}$

$\mathrm{H} 8 \mathrm{~A}-\mathrm{C} 8-\mathrm{H} 8 \mathrm{C}$

$\mathrm{H} 8 \mathrm{~B}-\mathrm{C} 8-\mathrm{H} 8 \mathrm{C}$

C14-C15-H15A

C14-C15-H15B

$\mathrm{H} 15 \mathrm{~A}-\mathrm{C} 15-\mathrm{H} 15 \mathrm{~B}$

$\mathrm{C} 14-\mathrm{C} 15-\mathrm{H} 15 \mathrm{C}$

$\mathrm{H} 15 \mathrm{~A}-\mathrm{C} 15-\mathrm{H} 15 \mathrm{C}$

H15B-C15-H15C

$\mathrm{C} 12-\mathrm{C} 16-\mathrm{H} 16 \mathrm{~A}$

$\mathrm{C} 12-\mathrm{C} 16-\mathrm{H} 16 \mathrm{~B}$

$\mathrm{H} 16 \mathrm{~A}-\mathrm{C} 16-\mathrm{H} 16 \mathrm{~B}$

$\mathrm{C} 12-\mathrm{C} 16-\mathrm{H} 16 \mathrm{C}$

$\mathrm{H} 16 \mathrm{~A}-\mathrm{C} 16-\mathrm{H} 16 \mathrm{C}$

$\mathrm{H} 16 \mathrm{~B}-\mathrm{C} 16-\mathrm{H} 16 \mathrm{C}$

$\mathrm{N} 1-\mathrm{C} 9-\mathrm{C} 14-\mathrm{C} 15$

$\mathrm{C} 14-\mathrm{C} 9-\mathrm{C} 10-\mathrm{C} 11$

$\mathrm{N} 1-\mathrm{C} 9-\mathrm{C} 10-\mathrm{C} 11$

$\mathrm{O} 1-\mathrm{C} 1-\mathrm{C} 2-\mathrm{C} 3$

$\mathrm{C} 6-\mathrm{C} 1-\mathrm{C} 2-\mathrm{C} 3$

$\mathrm{C} 1-\mathrm{C} 2-\mathrm{C} 3-\mathrm{C} 4$

$\mathrm{C} 5-\mathrm{C} 4-\mathrm{C} 3-\mathrm{C} 2$

$\mathrm{C} 8-\mathrm{C} 4-\mathrm{C} 3-\mathrm{C} 2$

$\mathrm{C} 9-\mathrm{C} 14-\mathrm{C} 13-\mathrm{C} 12$

$\mathrm{C} 15-\mathrm{C} 14-\mathrm{C} 13-\mathrm{C} 12$

$\mathrm{C} 11-\mathrm{C} 12-\mathrm{C} 13-\mathrm{C} 14$

$\mathrm{C} 16-\mathrm{C} 12-\mathrm{C} 13-\mathrm{C} 14$

$\mathrm{C} 9-\mathrm{C} 10-\mathrm{C} 11-\mathrm{C} 12$

$\mathrm{C} 13-\mathrm{C} 12-\mathrm{C} 11-\mathrm{C} 10$

$\mathrm{C} 16-\mathrm{C} 12-\mathrm{C} 11-\mathrm{C} 10$
$-0.5(3)$

123.48 (17)

118.3

118.3

121.27 (19)

119.4

119.4

109.5

109.5

109.5

109.5

109.5

109.5

109.5

109.5

109.5

109.5

109.5

109.5

109.5

109.5

109.5

109.5

109.5

109.5

$-1.2(3)$

-178.95 (16)

178.64 (16)

$-0.8(3)$

0.3 (3)

-0.1 (3)

$-179.56(17)$

$-2.0(3)$

$178.60(18)$

$0.6(3)$

$-177.52(18)$

-0.3 (3)

$0.6(3)$

178.71 (19)

Hydrogen-bond geometry $\left(A,{ }^{\circ}\right)$

$\mathrm{Cg} 2$ is the centroid of the $\mathrm{C} 9-\mathrm{C} 14$ ring.

\begin{tabular}{lllll}
\hline$D-\mathrm{H} \cdots A$ & $D-\mathrm{H}$ & $\mathrm{H} \cdots A$ & $D \cdots A$ & $D-\mathrm{H} \cdots A$ \\
\hline $\mathrm{O} 1-\mathrm{H} 1 \cdots \mathrm{N} 1$ & 0.82 & 1.89 & $2.618(2)$ & 147
\end{tabular}


Symmetry code: (i) $x+1 / 2,-y+1 / 2,-z$. 